\title{
Von der Krux wissenschaftlicher Politikberatung
}

\author{
Christoph Bertram*
}

I.

$\mathrm{D}$ ie Krux wissenschaftlicher Politikberatung rührt vor allem daher, dass Politik und Wissenschaft nun einmal schlecht zusammenpassen. Es sind zwei Welten mit grundverschiedenen Gestaltungszielen und Verfahren. Wissenschaftliche Politikberatung lebt in und aus diesem Spannungsverhältnis. Und weil sie ein Angebot an die Politik ist, von der Wissenschaft zu profitieren, muss diese sich weitgehend den Anforderungen und Aktionsweisen der Politik anpassen.

Dazu gehört eine Grunderkenntnis: Politik ist so faszinierend, weil sie die Fähigkeit zum Handeln bietet, anders als die Wissenschaft und der Journalismus. Aber weil jedes Handeln seinen Preis hat, zieht die Politik es vor, lieber nichts als etwas zu tun, und wenn doch, dann lieber weniger als viel.

Das gilt besonders für den demokratischen Staat im Innern und für die Zusammenarbeit der Staaten draußen in der Welt. Weil so viele mitspielen müssen, ist Blockieren die wirksamste Ausübung politischer Macht. Um doch zu einem Ergebnis zu gelangen, sind Kompromisse unumgänglich, ein für die Wissenschaft fremder, ja unstatthafter Begriff. Politik ist deshalb in der Regel handlungsscheu. Sie handelt, wie ein weiser ehemaliger Staatssekretär zu sagen pflegte, erst dann, wenn die Fingerkuppe des Kindes sich bereits vom Brunnenrand löst.

Das gilt erst recht in der Außen- und Sicherheitspolitik. Hier sind Situationen, in denen Regierungen schnell und mit vollem Einsatz handeln müssen, zum Glück selten geworden. Welche internationale Entwicklung, die Finanzkrise eingeschlossen, bedroht heute noch die Substanz unserer Sicherheit in einer Weise, die sofortiges Eingreifen der Politik verlangte? Natürlich lassen sich rasch zahlreiche mögliche Gefährdungen aufzählen, von der Instabilität fragiler Staaten zu Terrorismus und organsierter Kriminalität; und viele, die sich als wissenschaftliche Politikberater verstehen, verweisen gerne auf solche Gefahren, um die Politik zu Taten anzuspornen. Aber sie vergessen leicht, dass die Politik in solchen Handlungsempfehlungen eher das Risiko als die Chance sieht. Warum sollte sie dieses Risiko eingehen? Dann doch lieber abwarten. Von Winston Churchill stammt das verführerische Wort, das fast zum Verhaltenskodex der Politik geworden ist: A problem delayed is a problem half-solved - ein Problem, das man aufschieben kann, ist zur Hälfte gelöst.

Handlungsträgheit ist für Regierungen weise, so unerträglich sie für Politikberater auch sein mag. Die Politik reagiert lieber, als dass sie agiert. Es geht ihr mehr um Kontrolle als um Einfluss und Gestaltung - hierin liegt zum Beispiel auch die Lösung des andauernden Rätsels, warum europäische Regierungen es immer noch vorziehen, sich in der Welt als souveräne Staaten auszugeben, obgleich sie längst internationale Leichtgewichte geworden sind und nur in gemeinsamer europäischer Verbindung internationales Gewicht und internationalen Einfluss zustande brächten. In der Politik gilt, in leichter Abwandlung eines Reagan-Wortes, das die alten Rüstungskontrolleure noch erinnern werden: Einfluss ist schön und gut, aber Kontrolle ist besser.

Ein Prachtbeispiel für derartige Handlungsträgheit bietet die Außenpolitik der Bundesrepublik Deutschland; fast kann man sagen, ihre Außenpolitik besteht darin, keine zu haben. Wenigstens ist dies eine Vermutung, die sich bei all jenen einnisten muss, die von der deutschen Außenpolitik erwarten, sie solle endlich strategisch denken, deutsche Interessen formulieren und Prioritäten benennen. Aber hat das, was man für Trägheit oder Gestaltungsfaulheit halten mag, nicht vielleicht sogar Methode: Ist Deutschland, so könnte man zur Rechtfertigung von beidem vorbringen, in den letzten Jahrzehnten nicht ganz ordentlich damit gefahren - warum also etwas ändern und die Risiken des Handelns eingehen?

Wie anders erklärt sich die Berliner Neigung zu außenpolitisch punktueller Emsigkeit, kurzfristigen Placebos, die Abneigung nicht nur gegen militärische, sondern alle Interventionen, sofern es nicht um Handelsfragen geht? Wie soll man sonst die seit Jahren in unserer politischen Klasse - die Medien eingeschlossen - geübte Gleichgültigkeit gegenüber der europäischen Integration (und das kürzliche jähe Erwachen) und der atlantischen Zusammenarbeit deuten, die Ausdünnung außenpolitischer Fachkenntnis in unseren Parteien, Parlamenten, unseren Medien?

Gewiss, die Zahl außenpolitischer Fachleute jenseits der politischen Apparate ist in Deutschland rapide gewachsen. Aber wer meint, die Quantität an Kenntnissen würde sich in Qualität deutscher Außenpolitik oder doch in wachsendes außenpolitisches Interesse in der Öffentlichkeit umsetzen, wird rasch ernüchtert. In der Diskussion über die Wünschbarkeit eines deutschen Sitzes im UN-Sicherheitsrat argumentierte ein kluger Diplomat vor ein paar Jahren, damit würde auch das Bewusstsein internationaler Verantwortungsteilhabe in Deutschland wachsen. Aber auch hier geht es Berlin, wie wir erleben, nicht um Gestaltungsehrgeiz, sondern um Status.

II.

Allerdings braucht es nicht viel prophetische Gabe, um vorhersagen zu können, dass eine handlungsresistente Politik irgendwann scheitern muss. Die eine Hälfte von Churchills Problem 
mag sie durch Aussitzen gelöst haben, die andere dagegen verlangt aktive, kreative Gestaltung, wenn sie nicht zur wachsenden Sorge werden soll. Und dies anzustoßen ist die schwierige, aber schöne Aufgabe wissenschaftlicher Politikberatung.

Die Berufsbezeichnung ,Politikberater' ist nicht urheberrechtlich geschützt; in den Hauptstädten, auch unserer, wimmelt es nur so von Personen, die die Politik auf die eine oder andere Weise beraten wollen. Wissenschaftliche Politikberatung hat dagegen einen besonderen Anspruch, nämlich in geistiger Unabhängigkeit und mit wissenschaftlicher Erkenntnismethode Ergebnisse erarbeiten zu können, die gerade deshalb für die Politik nützlich sind, weil diese beides nicht aufbringen kann. Das gilt sehr allgemein. Aber was braucht die Politik wirklich an wissenschaftlicher Beratung von außerhalb des bürokratischen Apparats oder was sollte sie brauchen?

Am wenigsten wohl spezifische, aktuelle Informationen. Die produziert der bürokratische Apparat in der Regel verlässlich, rasch und in den für die politischen Entscheidungsträger nützlichen Portionen. Regierungen sind ausgestattet mit Spezialbehörden, Expertengremien, Nachrichtendiensten, diplomatischen Vertretungen, eingearbeiteten Referenten in den Ministerien - und alle produzieren unaufhörlich Informationen. Für den täglichen Normalbetrieb hat die Politik davon genug.

Was sie dagegen nicht selbst generieren kann und deshalb am meisten braucht, ist zweierlei: Evaluierungen bisheriger Leistung, einschließlich der Fehlerdiagnose, und vorausschauende Gesamtanalysen mit konkreten Handlungsempfehlungen. Die Evaluierung von Regierungs-handeln geschieht leider zu wenig, ein bisschen im Bereich der Entwicklungszusammenarbeit, am sichtbarsten etwa bei den jährlichen Gutachten der Wirtschaftsweisen. Im Übrigen bleibt die Überprüfung geleisteter Arbeit dem demokratischen Prozess und der Presse überlassen, aber beide sind zu systematischer Bewertung wenig geeignet.

Eine Ausweitung von externer Evaluierung wäre sicherlich wünschenswert, die Wissenschaft könnte hier ein nützlicher Partner sein. Nur ist die Schwierigkeit offenkundig: Wer verfügte gegenüber der Politik über die nötige Kompetenz, Information und Autorität, zumal in der Außen- und Sicherheitspolitik, um ihr präzise auf die Finger zu schauen und notfalls zu klopfen? Von wem würden die politischen Institutionen sich externe Einschätzungen gefallen lassen, wie sie für wissenschaftliche Institutionen längst selbstverständlich geworden sind?

Bleibt der zweite potenziell nützliche Beitrag wissenschaftlicher Beratung für die Politik: längerfristige Analysen, die über eine Legislaturperiode Bestand beanspruchen und konkrete Handlungsoptionen und -empfehlungen bieten. Diese Aufgabe ist leichter formuliert als verwirklicht. Das liegt nicht nur an den Gesetzmäßigkeiten des politischen Geschäfts, sondern auch an den Schwierigkeiten der Wissenschaft, ihre Verfahren, ihren Anspruch und ihre liebgewonnenen Gewohnheiten zugunsten des politischen Bedarfs zu ändern.

Einige dieser Schwierigkeiten sind offenkundig. Die Politik muss schnell und auf unsicherer Informationslage reagieren und oft unter lauter schlechten Optionen eine auswählen; Henry Kissinger hat einmal bemerkt, dass richtige politische Entscheidungen in der Regel zu 49 Prozent falsch sind. Für die Wissenschaft wäre so etwas undenkbar. Sie sucht in Ruhe und Abgeschiedenheit die richtige Erkenntnis und will nichts abliefern, bevor die Arbeit getan ist. Wo die Politik kurze und schnelle Stellungnahmen will, sucht die Wissenschaft gründliche Auseinandersetzung, die durch Vorgaben für Ablieferungstermin und Präsentationsumfang nicht eingezwängt werden darf.

Für die Politik ist jedes Ereignis ein Einzelfall, für die Wissenschaft am liebsten Symptom für eine darüber hinaus gültige Erklärung, eine Theorie. Und da auch die Politologen richtige Wissenschaftler sein wollen, haben sie sich eifrig in den Theorie-Dschungel begeben und genießen ihren eigenen Theoriestreit als Bestätigung. So verständlich allerdings der Wunsch, so fragwürdig seine Umsetzung angesichts der Vielfalt und Komplexität politischer Prozesse.

Das gilt im internationalen Bereich erst recht. Hier explodiert die Zahl der Akteure, die Erklärungsmuster werden immer relativer. ${ }^{1}$ Aber für unser Thema ist nicht entscheidend, ob die Politik theoriegeeignet ist, sondern ob sie mit den Theorien etwas anfangen kann. Und hier ist die Antwort erst recht negativ.

Diese Aufzählung der Kluft zwischen dem politischen und dem wissenschaftlichen Prozess soll keine unfaire Kritik an der Wissenschaft sein. Wenn der Ansatz beider gleich wäre, bedürfte es keiner Politikberatung mit wissenschaftlichem Anspruch. Es sind ja gerade die dem Politikbetrieb fremden Eigenschaften der Wissenschaft, welche ihrem Input Gewicht und Autorität geben.

Wissenschaftliche Politikberatung kann Politik besser und wirksamer machen, gerade weil sie einem anderen Zeitrhythmus folgt, politischen Sachzwängen und dem Konkurrenzdruck politischer Kräfte nicht unterliegt und nicht von politischen Ideologien bestimmt ist.

Aber gerade deshalb ist es so wichtig, sich der Kluft bewusst zu sein. Denn nur, wenn die wissenschaftliche Politikberatung wenigstens einen Teil dieser Kluft überbrücken kann, wird sie wirksam werden. Tut sie es nicht, wird sie zu Recht in den Archiven des Elfenbeinturms abgelegt.

\section{III.}

Wie kann Wissenschaft am besten die Politik beraten, ohne ihr wissenschaftliches Selbstverständnis aufzugeben?

Sie muss vor allem lernen, sich auf die Gegebenheiten und Funktionsumstände ihres Kunden einzustellen. Wissenschaftliche Politikberatung ist Dienst am Kunden Politik, nicht am Kunden Wissenschaft! Das sich Hineindenken in den Bedarf der Politik ist dafür die wichtigste mentale Voraussetzung. Es kann deshalb nur nützen, wenn der Wissenschaftler immer

1 In meinen Jahren als Forscher, Forschungsmanager, Leiter von Forschungsinstituten und temporärer akademischer Lehrer habe ich die Leidenschaft der IR-Wissenschaftler für die theoretische Auseinandersetzung nie aus der Theorie-Eignung des Materials erklären können. Vielmehr erscheinen mir die Theorien entweder als so vage, dass sie auf alles passen und deshalb wenig bringen, oder als so ehrgeizig, dass sie eine andere politische Realität voraussetzen. 
wieder eine Zeit im politischen Apparat verbringt, bevor er wieder an seinen Schreibtisch zurückkehrt.

Hineindenken bedeutet nun nicht, zum bloßen Handlanger des politischen Auftraggebers zu werden. Denn oft weiß auch dieser Kunde nicht, was er wirklich braucht, erst die Wissenschaft kann ihm helfen, es zu entdecken. Eben deshalb ist weniger die Detailkunde gefragt als der Überblick, der die Verknüpfungen der verschiedenen Elemente erkennt, die jedem politischen Problem eigen sind, und sie berücksichtigt. Die Politik, um es kurz zu sagen, braucht weniger den Spezialisten als den gebildeten Generalisten.

Dabei muss der wissenschaftliche Berater immer eine Antwort suchen, die aus der Sicht des zu Beratenden Sinn ergibt. In der Außen- und Sicherheitspolitik ist das die Frage, inwieweit die nationalen Interessen berührt sind und wie sie am besten verfolgt werden können. Länderkunde, also die Spezialisierung auf ein bestimmtes, noch dazu möglichst fernes Land, ohne jeden Bezug auf die Auswirkungen für deutsches Handeln hilft der Politik nicht weiter, auch wenn sich immer mehr Forscherinnen und Forscher in Einrichtungen wissenschaftlicher Politikberatung damit befassen.

Ebenso wenig hilft es politischen Entscheidungsträgern, wenn die Berater ihnen Handlungen nahebringen, ohne die politischen Realitäten zu beachten. Das unter fortschrittlichen Politologen so beliebte Konzept der „Responsibility to protect“ ist dafür ein herausragendes Beispiel. Keine Regierung wird sich einen Interventionsautomatismus abverlangen lassen, nur um diesem Konzept gerecht zu werden. Sie wird sich höchstens in Einzelfällen, von denen keiner ein Präzedenzfall sein darf, unter großen Vorbehalten zum Einsatz militärischer oder anderer Zwangsmittel entschließen.

Das mag sie dann mit so schönen Formeln wie der von einer internationalen Schutzverantwortung drapieren, aber immer mit der Mentalreservation, sich dadurch nicht für die Zukunft zu binden. Es wäre ja auch verwunderlich, wenn es anders wäre immerhin muss ja die Regierung, nicht der Berater, für die Folgen einstehen, und die können für manche tödlich sein. Verwunderlich ist allenfalls die Neigung vieler Wissenschaftler, immer noch zu glauben, es wäre anders.

Dienst am Kunden heißt schließlich, nach den weichen Stellen in der Außenhaut des politischen Apparats zu suchen, an denen wissenschaftliche Beratung eine bessere Chance hat zum Verbraucher zu gelangen. Zwei sind es vor allem: die Arbeitsebene in Ministerien und die Parlamentarier.

Die Spitze hat genügend Berater. Aber die Referenten, welche die Vorlagen für die Spitze verfassen, sind in aller Regel dankbar, wenn Analysen mit wissenschaftlichem Gewicht auf ihren Schreibtisch kommen - vorausgesetzt, sie sind hinreichend kurz und bündig. Referenten sind für Beratung zugänglicher als Abteilungsleiter, von Ministern gar nicht zu sprechen. Auch Parlamentarier sind für Beratung aufgeschlossen, weil sie ohnehin bürgernäher sind und über weniger Informationen verfügen als der Regierungsapparat.

Aber auch wer all diese Regeln beachtet, sich in Bedarf und Handlungsumstände der Politik hineindenkt, Zusammenhänge aufdeckt statt nur die punktuelle Emsigkeit der Bürokratie zu verstärken, den knappen Zeithaushalt seiner Kunden nicht durch Langatmigkeit oder theoretische Ausflüge überstrapaziert - auch wer dies alles beherzigt, der wird selten feststellen, dass sich sein Rat in späteren Entscheidungen der Exekutive wiederfindet.

Mit Politikberatung, gerade der wissenschaftlich fundierten, kann man nicht angeben, Aha-Erlebnisse sind selten, eine 1:1Umsetzung von Rat in Tat ist praktisch ausgeschlossen. Der Berater muss sich schon glücklich schätzen, wenn seine Expertise irgendwie einfließt in den Strom von vielen anderen Inputs. Wenn ein Politikberater behauptet, eine bestimmte Entscheidung sei seinem Rat zu verdanken, ist Misstrauen angebracht ganz abgesehen davon, dass er wohl bald arbeitslos sein wird, weil Politiker es wenig schätzen, wenn sich jemand damit brüstet, ihnen den Weg gewiesen zu haben.

Deshalb ist wissenschaftliche Politikberatung in der Regel nur indirekte Mitwirkung am politischen Konsensprozess. Aber wäre das wirklich ein unerträglich mageres Ergebnis? Das Hineinfließen von vielen Positionen und Beurteilungen in das Meinungsklima eines Landes, einer Regierung, einer Partei entspricht dem normalen demokratischen Prozess. Vom Journalismus hat Gertrude Stein, der literarische Andy Warhol ihrer Zeit, einmal gesagt, er sei wie Regentropfen, die auf die Oberfläche des Ozeans fallen. Mit der wissenschaftlichen Politikberatung ist es wenig anders: Auch sie kann, richtig eingesetzt, Kontext und Temperatur auf dem politischen Ozean beeinflussen und den Salzgehalt etwas reduzieren, nicht mehr, aber auch nicht minder.

Das politische Meinungsklima schafft den Kontext, in den politische Entscheidungen sich einfügen wollen, es ergibt eine Messlatte, nach der die Beiträge zur demokratischen Debatte gewertet werden. Es ist der Nährboden für den politischen Konsens. Weil dieser die öffentliche Debatte verlangt, muss wissenschaftliche Politikberatung, von vertraulichen Gutachten abgesehen, immer auch die Öffentlichkeit einbeziehen.

Die Mitwirkung an der Gestaltung des politischen Meinungsklimas ist denn auch die schönste Rolle wissenschaftlicher Politikberatung. Sie mag oft ein Kreuz sein. Aber es lohnt sich, es zu tragen. 